\title{
Optimal Tracking Performance of MIMO Discrete-Time Systems with Network Parameters
}

\author{
Chao-Yang Chen, ${ }^{1,2}$ Weihua Gui, ${ }^{2}$ Zhi-Hong Guan, ${ }^{3}$ Shaowu Zhou, ${ }^{1}$ and Cailun Huang ${ }^{1}$ \\ ${ }^{1}$ School of Information and Electrical Engineering, Hunan University of Science and Technology, Xiangtan 411201, China \\ ${ }^{2}$ School of Information Science and Engineering, Central South University, Changsha 410012, China \\ ${ }^{3}$ College of Automation, Huazhong University of Science and Technology, Wuhan 430074, China \\ Correspondence should be addressed to Chao-Yang Chen; ouzk@163.com
}

Received 23 March 2016; Revised 21 July 2016; Accepted 26 July 2016

Academic Editor: Guoqiang Hu

Copyright ( $) 2016$ Chao-Yang Chen et al. This is an open access article distributed under the Creative Commons Attribution License, which permits unrestricted use, distribution, and reproduction in any medium, provided the original work is properly cited.

\begin{abstract}
The optimal regulation properties of multi-input and multioutput (MIMO) discrete-time networked control systems (NCSs), over additive white Gaussian noise (AWGN) fading channels, based on state space representation, are investigated. The average performance index is introduced. Moreover, the regulation performance is measured by the control energy and the error energy of the system, and fundamental limitations are obtained. Two kinds of network parameters, fading and the additive white Gaussian noise, are considered. The best attainable regulation performance limitations can be obtained by the limiting steady state solution of the corresponding algebraic Riccati equation (ARE). The simulation results are given to demonstrate the main results of the theoretical development.
\end{abstract}

\section{Introduction}

In recent years, there has been growing attention devoted to the study of feedback control over communication networks [1-9], because, comparing with classical feedback control systems, the NCSs have their advantages, for example, low cost, flexibility, reduced weight and power requirement, and simple installation and maintenance. However, there exist many tough challenging problems in the stability and performance analysis of NCSs owing to the existence of networks. Researchers keep their eyes on the communication constraints in the networks, for example, quantization effects $[10,11]$, time delay [12-15], data rate constraint $[16,17]$, and data packet dropout $[12,18,19]$. Nevertheless, the performance limitation of NCSs remains a puzzle.

Performance limitation of control systems has been receiving an increasing amount of interest in the control community; see [20-24] for details. A partial review of previous work on feedback performance over a communication channel is given as follows. By invoking Shannon entropy as a measure of performance, a universal lower bound was obtained in [25]. Reference [26] derived a conservation law dictating that causal feedback cannot reduce the differential entropy inserted in the loop by external sources and an inequality unveiling that the feedback loop must be able to convey information originating from initial states of the physical plant and exogenous disturbance signals. By using nonlinear timevarying communication and control strategies, [27] proposed a lower bound on the performance achievable at a specified terminal time and pointed out that the bound can be achieved by linear strategies. Reference [28] showed the performance limitations for scalar systems under either bounded or Gaussian disturbances, and two kinds of disturbances were treated in a unified manner using appropriate entropies and distortions. However, in [28], the achievable performance had not been improved even if the maximum information constraint is relaxed to an average information constraint. Optimal tracking performance issues were studied for multi-input and multioutput linear time-invariant systems under networked control with limited bandwidth and additive colored white Gaussian noise channel in [22]. In [1], the optimal tracking performance of NCSs with encoder-decoder was studied. The optimal tracking performance of single-input single-output (SISO) discrete-time NCSs with the packet dropouts and 
channel noise is studied in [2]. The communication channel is characterized by three parameters: the packet dropouts, channel noise, and the encoding and decoding. In [3], the optimal tracking performance of MIMO discrete-time NCSs with bandwidth and coding constraints is studied by using spectral factorization technique. In [4], the limitations in stabilization and tracking of MIMO networked feedback systems are studied. The reference is considered as a random reference signal with finite power. The optimal tracking performance by linear time-invariant (LTI) controllers subject to channel input power constraint is obtained. The adopted model can be found in many real systems. For example, in the telemedicine system of robot-assisted neurosurgery, patient and robot are, respectively, the plant and the controller. The remote expert obtains information via the network transmission, and the instruction of the expert is then sent back to the robot via the network transmission. In addition, for leader-follower multiagent systems [29], provided that the position, velocity, and direction information of a leader are considered as the reference signal, the controller is designed to achieve the minimal tracking error between the leader and the follower.

In this paper, we investigate optimal regulation performance issues pertaining to MIMO feedback control systems over multiple AWGN fading channels. The average performance index is introduced, and the regulation performance is measured by controlling energy and the state energy of the system. And regulation fundamental limitations are obtained. The stability or stabilization problem for the network with a fading channel is considered in a few works [30, 31]. Moreover, few results about the performance limitation analysis of the network with a fading channel can be found nowadays. Due to the impact of multiplicative noise in fading channel, it is difficult to be processed and analyzed for the performance limitation with fading channels by the frequency domain method. Therefore, in this paper, the performance limitation is considered from another angle, that is, the state space method. Additionally, in most of the existing results, the best achievable performance is analyzed under transfer function representation $[1-4,21,22,26,32]$. However, from a modern control theoretic point of view, this is not the only possible line of research to pursue. The goal of this paper is to derive the regulation performance under state space representation. The contributions of this paper can be summarized as follows. Firstly, the model about multiple AWGN fading channels is considered, which is more practical than most existing literatures focusing on AWGN channel models, for instance, $[4,21,22]$. Secondly, we are mainly devoted to study the performance limitations for the NCS with fading channels and AWGN, which is different from the results $[30,31]$ of the existing focus on the stability or stabilization problem for the NCS with fading channel. Furthermore, the best attainable regulation performance limitations can be reached by the limiting steady state solution of its associated algebraic Riccati equation (ARE).

The rest of the paper is organized as follows. The feedback regulation performance limitations are studied by parameter

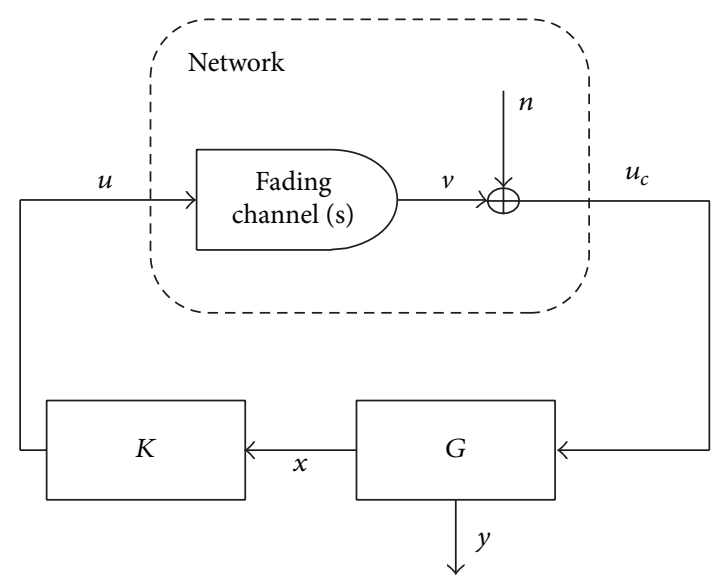

FIGURE 1: State feedback control by one-parameter controller over AWGN fading channels.

controller in Section 2. Simulation studies are shown to validate the theoretical results in Section 3. Concluding remarks are made in Section 4.

Terminology. The open unit disk is denoted by $\mathbb{D}$, the closed unit disk by $\overline{\mathbb{D}}$, and their complements by $\mathbb{D}^{C}$ and $\overline{\mathbb{D}}^{C}$, respectively. $M^{T}$ is the transpose of a matrix $M$ and $M^{\dagger}$ is its Moore-Penrose pseudo inverse; and $\operatorname{Tr}(M)$ is the trace of a square matrix $M . E[x]$ represents the expectation of a random variable $x$.

\section{Regulation Performance Limitations}

In this work, we will consider a feedback control system with a network in the upstream channel as showed in Figure 1, where the plant model $G$ is a rational transfer function matrix. The network model is an unreliable network in the path from the controller to the plant, which contains a fading channel and an additive white Gaussian noise (AWGN) channel.

Assume that the plant $G(s)$ is strictly proper and unstable, and one of its minimum realizations is given by

$$
\begin{aligned}
x(k+1) & =A x(k)+B u_{c}(k), \\
y(k) & =C x(k),
\end{aligned}
$$

where $x(k) \in \mathbb{R}^{p}, u_{c}(k) \in \mathbb{R}^{m}$, and $y(k) \in \mathbb{R}^{p}$ are the plant state, the plant input, and the measured output, respectively. Obviously, $A$ is unstable, $B=\left[\begin{array}{llll}B_{1} & B_{2} & \cdots & B_{m}\end{array}\right]$ has fullcolumn rank, $C=\left[\begin{array}{llll}C_{1}^{T} & C_{2}^{T} & \cdots & C_{p}^{T}\end{array}\right]^{T}$ has full-row rank, and the triple $(A, B, C)$ is stabilizable and detectable.

The input and output relationship for the AWGN fading channel is given by

$$
u_{c}(k)=v(k)+n(k)
$$

where $v(k)$ is the fading channel output and $n(k)=\left(n_{1}(k), \ldots\right.$, $\left.n_{m}(k)\right)^{T}$ is a vector of uncorrelated zero-mean white noises each with power spectral density $\Phi_{i}(k), 1 \leq i, k \leq m$, for each element and $\Phi=\operatorname{diag}\left\{\Phi_{1}(k), \ldots, \Phi_{m}(k)\right\}$. And $v(k)$ and 
$n(k)$ are independent of each other. The model of the fading channel is given in the following memoryless multiplicative form:

$$
v(k)=\zeta(k) u(k)
$$

where $u(k)$ is the network control input, and

$$
\zeta(k)=\left\{\zeta_{1}(k) \zeta_{2}(k) \cdots \zeta_{m}(k)\right\},
$$

with $\left\{\zeta_{i}(k)\right\}$ being $m$ independent random variables at each time index $k$. And it is assumed that $\left\{\zeta_{i}(k)\right\}$ are white noise processes with

$$
\begin{aligned}
\mu_{i} & :=E\left\{\zeta_{i}(z)\right\}, \\
\sigma_{i}^{2} & :=E\left\{\left(\zeta_{i}(z)-\mu_{i}\right)^{2}\right\},
\end{aligned}
$$

satisfying $\mu_{i}>0, \sigma_{i}>0, i=1,2, \ldots, m$. Denote

$$
\begin{aligned}
& \Pi:=\operatorname{diag}\left\{\mu_{1}, \mu_{2}, \ldots, \mu_{m}\right\}, \\
& \Lambda:=\operatorname{diag}\left\{\sigma_{1}, \sigma_{2}, \ldots, \sigma_{m}\right\} .
\end{aligned}
$$

Remark 1. Besides the fading phenomenon, the uncertainties of a digital network can be described by the model (3), such as packet dropouts and quantization errors [30]. Specifically, the model (3) covers packet dropout described by identically and independently distributed (i.i.d.) Bernoulli processes [30]. The $\zeta(k)$ represents the packet-loss process by a $0-1$ binaryvalued scalar.

The following lemmas are useful for subsequent development and thus are introduced first.

Lemma 2 (see [31]). G is a nonminimum phase strictly proper right-invertible transfer function matrix; then, a state space realization of $G$ can be given by

$$
\begin{aligned}
& A=\left[\begin{array}{cc}
A_{s} & 0 \\
0 & A_{u}
\end{array}\right], \\
& B=\left[\begin{array}{l}
B_{s} \\
B_{u}
\end{array}\right], \\
& C=I, \\
& D=0,
\end{aligned}
$$

where $A_{s}$ is stable, all the poles of $A_{u}$ are either on or outside the unit circle, and $\left(A_{u}, B_{u}\right)$ is controllable.

Lemma 3 (see [33]). The equation $A X B=C$ has a solution $X$ if and only if

$$
A A^{\dagger} C B^{\dagger} B=C
$$

Moreover, the general solution is

$$
X=A^{\dagger} C B^{\dagger}+Y-A^{\dagger} A Y B B^{\dagger},
$$

where $Y$ is arbitrary.
2.1. State Feedback Regulation Performance Limitations. In this subsection, we consider the feedback system of Figure 1 where the channel input

$$
u(i)=-K_{i} x(i)
$$

is based on static state feedback. To simplify the form, we recorded $u(i):=u_{i}$. The average performance index to be minimized in the present subsection is given by

$$
\begin{aligned}
J= & \lim _{N \rightarrow \infty} \frac{1}{N} \inf _{u_{i} \in U_{\mathrm{sf}}} J\left(x_{0}, u_{0}, u_{1}, \ldots, u_{N}\right) \\
= & \lim _{N \rightarrow \infty} \frac{1}{N} \inf _{u_{i} \in U_{\mathrm{sf}}} E\left\{\sum_{i=0}^{N}\left(\varepsilon\left\|y_{i}\right\|^{2}+(1-\varepsilon)\left\|u_{i}\right\|^{2}\right)\right\} \\
= & \lim _{N \rightarrow \infty} \frac{1}{N} \\
& \cdot \inf _{u_{i} \in U_{\mathrm{sf}}} \sum_{i=0}^{N} \operatorname{Tr}\left\{\left(Q+K_{i}^{T} R_{i} K_{i}\right) X_{i}+Q_{N+1} X_{N+1}\right\},
\end{aligned}
$$

where $\varepsilon \in[0,1]$ is a parameter to be determined prior to one's choice, and it may be used to weigh the relative importance of tracking objective versus that of constraining the input energy. $Q=\varepsilon C^{T} C, R=(1-\varepsilon) I, X_{i}=E\left(x_{i} x_{i}^{T}\right)$, and $Q_{N+1}$ is a symmetric positive definite matrix of appropriate dimension. $U_{\text {sf }}$ is the class of all stabilizing state feedback controllers.

The problem under study can be described as follows.

Problem 4. For a discrete-time NCS as depicted in Figure 1, find a network control input $u \in U_{\text {sf }}\left(K_{i}\right)$ such that the minimum performance index (11) is obtained.

Theorem 5. Consider the feedback system of Figure 1 with a fading channel by (2) and (3), and the system $G(z)$ is unstable, nonminimum phase, and strictly proper, $(A, B, C)$ is the minimum state space realization of $G(z)$, and then the minimum state regulation performance is given by

$$
J^{*}=\operatorname{Tr}\left\{B \Phi B^{T} P\right\}
$$

where $P$ is the unique solution of discrete-time ARE:

$$
P=Q+A^{T} P A-N^{T} M^{\dagger} N
$$

and the optimal controller sequence is

$$
u_{k}^{*}=-M^{\dagger} N x_{k}
$$

where

$$
\begin{aligned}
& M=R+\Pi B^{T} P B \Pi+\Lambda B^{T} P B \Lambda, \\
& N=\Pi B^{T} P A .
\end{aligned}
$$

Proof. The optimal problem is formulated in terms of the state covariance matrices $X_{i}=E\left[x_{i} x_{i}^{T}\right]$ and the gain matrices $K_{i}$. By a simple calculation it can be seen that the following deterministic optimal control problem is equivalent to the 
original problem (1), (2), (3), (10), (11), and (13), with a feedback control of the form (10):

$$
\begin{aligned}
J= & \lim _{N \rightarrow \infty} \frac{1}{N} \\
& \cdot \inf _{u_{i} \in U_{\mathrm{sf}}} \sum_{i=0}^{N} \operatorname{Tr}\left\{\left(Q+K_{i}^{T} R_{i} K_{i}\right) X_{i}+Q_{N+1} X_{N+1}\right\},
\end{aligned}
$$

subject to

$$
\begin{aligned}
X_{k+1}= & \left(A+B \Pi K_{k}\right) X_{k}\left(A+B \Pi K_{k}\right)^{T} \\
& +B \Lambda K_{k} X_{k} K_{k}^{T} \Lambda B^{T}+B \Phi B^{T} .
\end{aligned}
$$

Firstly, consider the following performance index:

$$
J_{N}:=\inf _{u_{i} \in U_{\text {sf }}} \sum_{i=0}^{N} \operatorname{Tr}\left\{\left(Q+K_{i}^{T} R_{i} K_{i}\right) X_{i}+Q_{N+1} X_{N+1}\right\} .
$$

In order to obtain the optimal performance $J_{N}^{*}$ under conditions (19), we can construct the following Lagrangian function:

$$
L=\sum_{i=0}^{N} H_{i}+Q_{N+1} X_{N+1},
$$

where $H_{i}$ is Hamiltonian function:

$$
\begin{aligned}
H_{i} & \triangleq \operatorname{Tr}\left[\left(Q+K_{i}^{T} R K_{i}\right) X_{i}\right]+\operatorname{Tr}\left[P _ { i + 1 } \left(A X_{i} A^{T}\right.\right. \\
& +B \Pi K_{i} X_{i} A^{T}+A X_{i} K_{i}^{T} \Pi B^{T}+B \Pi K_{i} X_{i} K_{i}^{T} \Pi B^{T} \\
& \left.\left.+B \Lambda K_{i} X_{i} K_{i}^{T} \Lambda B^{T}+B \Phi B^{T}-X_{i+1}\right)\right],
\end{aligned}
$$

where $P_{i+1}$ is a parameter matrix and $P_{i+1}$ is symmetric matrices of Lagrangian multiplier [34]. The necessary conditions for optimality are

$$
\begin{aligned}
\frac{\partial H_{i}}{\partial K_{i}} & =0, \\
P_{i} & =\frac{\partial H_{i}}{\partial X_{i}}, \\
P_{N+1} & =Q_{N+1} .
\end{aligned}
$$

Then, we can obtain the following AREs:

$$
\begin{aligned}
(R+ & \left.\Pi B^{T} P_{i+1} B \Pi+\Lambda B^{T} P_{i+1} B \Lambda\right) K_{i}+\Pi B^{T} P_{i+1} A \\
= & 0 \\
P_{i} & \\
= & Q+A^{T} P_{i+1} A \\
& +K_{i}^{T}\left(R+\Pi B^{T} P_{i+1} B \Pi+\Lambda B^{T} P_{i+1} B \Lambda\right) K_{i} \\
& +A^{T} P_{i+1} B \Pi K_{i}+K_{i}^{T} \Pi B^{T} P_{i+1} A, \\
P_{N+1} & =Q_{N+1} .
\end{aligned}
$$

And then, we have

$$
J_{N}^{*}=\operatorname{Tr}\left[X(0) P(0)+\sum_{i=0}^{N} B \Phi B^{T} P(i+1)\right] .
$$

It is known that the general finite ARE has a unique solution $P_{N}(t)>0, t \in\{0,1,2, \ldots, N\}$. It is obvious that $P_{N}(t)=$ $P_{N-t}(0)$. If the system (1) is stable by feedback control $u_{i}, i \in\{1,2, \ldots, N, \ldots, \infty\}$, the corresponding regulation performance limitation must exist; namely, $P(0)$ exists in (25), and the solution of general ARE (24) $\lim _{N \rightarrow \infty} P_{N}(0)$ exists. Moreover

$$
\lim _{N \rightarrow \infty} P_{N}(0)=\lim _{N \rightarrow \infty} P_{N-t}(0)=\lim _{N \rightarrow \infty} P_{N}(t)=P .
$$

Then, (24) can be written as

$$
\begin{aligned}
(R+ & \left.\Pi B^{T} P B \Pi+\Lambda B^{T} P B \Lambda\right) K_{i}+\Pi B^{T} P A=0, \\
P & \\
= & Q+A^{T} P A+K_{i}^{T}\left(R+\Pi B^{T} P B \Pi+\Lambda B^{T} P B \Lambda\right) K_{i} \\
& +A^{T} P B \Pi K_{i}+K_{i}^{T} \Pi B^{T} P A .
\end{aligned}
$$

Now by using Lemma 3, we can obtain

$$
K_{i}=-M^{\dagger} N+Y-M^{\dagger} M Y,
$$

$$
M M^{\dagger} N=N \text {, }
$$

where

$$
\begin{aligned}
& M=R+\Pi B^{T} P B \Pi+\Lambda B^{T} P B \Lambda, \\
& N=\Pi B^{T} P A .
\end{aligned}
$$

In particular, we can take $Y=M^{\dagger} N$ and noting (13), (20), (25), and (27), then the optimal performance, the control gain $K_{i}$, and the discrete-time ARE can be given by

$$
\begin{aligned}
K_{i} & =-M^{\dagger} N, \\
J_{\mathrm{opt}} & =\operatorname{Tr}\left\{B \Phi B^{T} P\right\}, \\
P & =Q+A^{T} P A-N M^{\dagger} N .
\end{aligned}
$$

Remark 6. Considering the traditional sense of the performance index

$$
\begin{aligned}
J & =\inf _{u_{i} \in U_{\text {sf }}} J\left(x_{0}, u_{0}, u_{1}, \ldots, u_{N}\right) \\
& =\inf _{u_{i} \in U_{\text {sf }}} E\left\{\sum_{i=0}^{N}\left(\varepsilon\left\|y_{i}\right\|^{2}+(1-\varepsilon)\left\|u_{i}\right\|^{2}\right)\right\},
\end{aligned}
$$

by the proof of Theorem 5, we can get that the performance limitation is

$$
J^{*}=\operatorname{Tr}\left[X(0) P(0)+\sum_{i=0}^{\infty} B \Phi B^{T} P(i+1)\right] .
$$


Noting (26), the performance limitation (32) will reach an infinite value for $P>0$, so we introduced the average performance limitations (11).

When the unreliable network does not contain the fading channel, that is, it contains only a white Gaussian noise channel, we can obtain the following corollary.

Corollary 7. Consider the feedback system of Figure 1 with a white Gaussian noise channel, and the system $G(s)$ is unstable and nonminimum phase corresponding to Lemma 2; then the minimum state regulation performance is given:

$$
J^{*}=\operatorname{Tr}\left\{B_{u} \Phi B_{u}^{T} P_{u}\right\}
$$

where $P_{u}$ is the unique solution of discrete-time ARE:

$$
P_{u}=Q_{u}+A_{u}^{T} P_{u} A_{u}-N_{u}^{T} M_{u}^{\dagger} N_{u} .
$$

And the optimal controller sequence is

$$
u_{k}^{*}=\left[\begin{array}{ll}
0 & -M_{u}^{\dagger} N_{u}
\end{array}\right] x_{k},
$$

where

$$
\begin{aligned}
& M=R+\Pi B_{u}^{T} P_{u} B_{u} \Pi+B_{u}^{T} P_{u} B_{u}, \\
& N=B_{u}^{T} P_{u} A_{u} .
\end{aligned}
$$

Proof. Similar to the previous section

$$
\begin{aligned}
J_{N} & :=\inf _{u_{i} \in U_{\mathrm{sf}}} \sum_{i=0}^{N} \operatorname{Tr}\left\{\left(Q+C^{T} K_{i}^{T} R_{i} K_{i} C\right) X_{i}+Q_{N+1} X_{N+1}\right\}, \\
L & :=\sum_{i=0}^{N} H_{i}+Q_{N+1} X_{N+1}, \\
H_{i} & :=\operatorname{Tr}\left[\left(Q+K_{i}^{T} R K_{i}\right) X_{i}\right]+\operatorname{Tr}\left[P _ { i + 1 } \left(A X_{i} A^{T}\right.\right. \\
& +A X_{i} K_{i}^{T} B^{T}+B K_{i} X_{i} A^{T}+B K_{i} X_{i} K_{i}^{T} B^{T}+B \Phi B^{T} \\
& \left.\left.-X_{i+1}\right)\right] .
\end{aligned}
$$

Then

$$
J_{N}^{*}=\operatorname{Tr}\left[X(0) P(0)+\sum_{i=0}^{N} B \Phi B^{T} P(i+1)\right] .
$$

And then, the following equations can be obtained:

$$
\begin{aligned}
(R+ & \left.B^{T} P B\right) K_{i}+B^{T} P A=0, \\
P & \\
= & Q+A^{T} P A+K_{i}^{T}\left(R+B^{T} P B\right) K_{i}+A^{T} P B K_{i} \\
& +K_{i}^{T} B^{T} P A .
\end{aligned}
$$

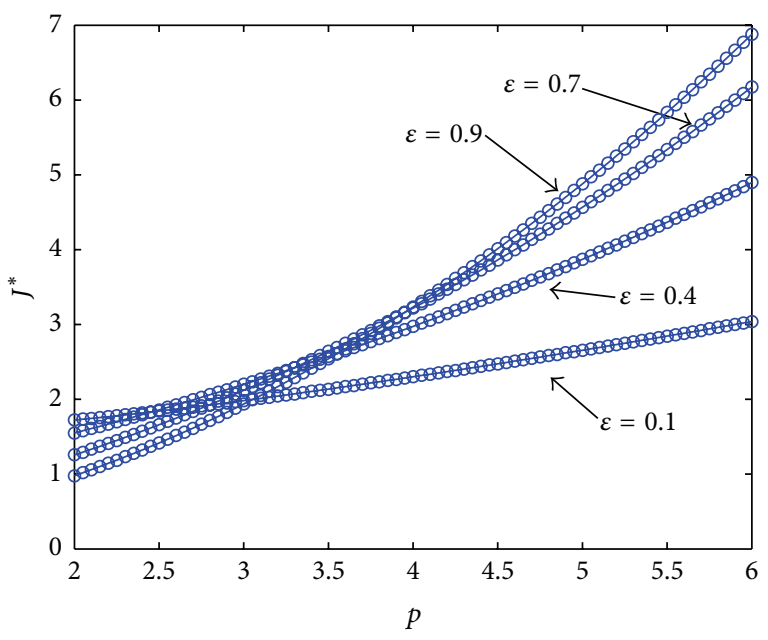

Figure $2: J^{*}$ with respect to $p$ for different $\varepsilon(q=1.8, \Phi=0.2$, $\Pi=0.6$, and $\Lambda=0.6$ ).

Applying Lemma 2, the above equation can be transformed into

$$
\begin{aligned}
(R+ & \left.B_{u}^{T} P B_{u}\right) K_{u}+B_{u}^{T} P_{u} A_{u}=0, \\
P_{u} & \\
\quad & Q_{u}+A_{u}^{T} P_{u} A_{u}+K_{u}^{T}\left(R+B_{u}^{T} P_{u} B_{u}\right) K_{u} \\
& \quad+A_{u}^{T} P_{u} B_{u} K_{u}+K_{u}^{T} B_{u}^{T} P_{u} A_{u} .
\end{aligned}
$$

Therefore, we have

$$
\begin{aligned}
& K_{i}^{*}=-M_{u}^{\dagger} N_{u}, \\
& J_{\mathrm{opt}}=\operatorname{Tr}\left\{B_{u} \Phi B_{u}^{T} P_{u}\right\}, \\
& P_{u}=Q_{u}+A_{u}^{T} P_{u} A_{u}-N_{u}^{T} M_{u}^{\dagger} N_{u},
\end{aligned}
$$

where

$$
\begin{aligned}
& M=R+\Pi B_{u}^{T} P_{u} B_{u} \Pi+B_{u}^{T} P_{u} B_{u}, \\
& N=B_{u}^{T} P_{u} A_{u} .
\end{aligned}
$$

\section{Simulation Studies}

We now use an example to illustrate Theorem 5. Consider the plant

$$
G(z)=\frac{(z-p)}{(z-1.5)(z-q)} .
$$

Clearly, $G(z)$ is nonminimum phase and unstable for $|p| \geq 2$ and $|q| \geq 1.5$.

Figures 2 and 3 show the optimal performances plotted for different values of $\varepsilon$. The two figures also show that the 


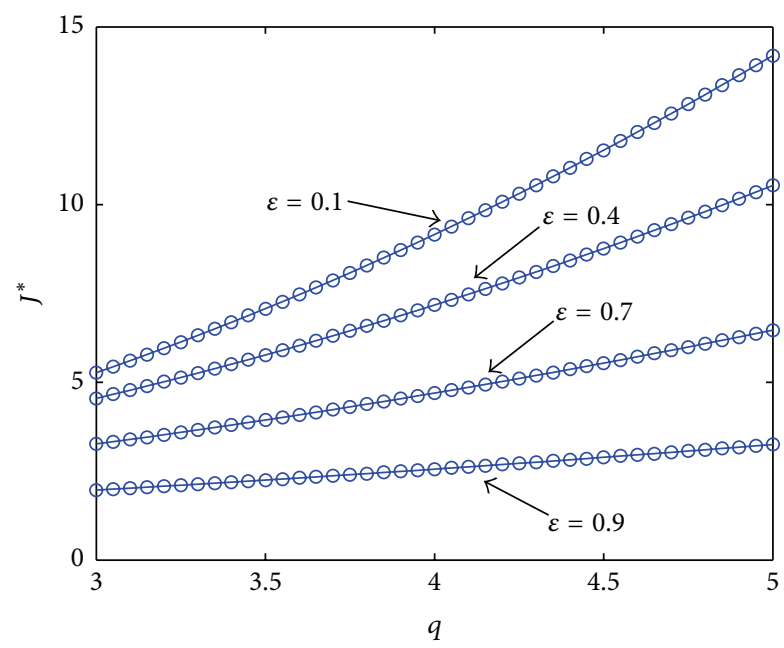

Figure 3: $J^{*}$ with respect to $q$ for different $\varepsilon(p=-2, \Phi=0.2$, $\Pi=0.6$, and $\Lambda=0.6$ ).

unstable poles/nonminimum phase zero will deteriorate the optimal regulation performances.

\section{Conclusions}

In this paper, we have investigated the optimal regulation performance of networked control systems over an unreliable network in the path from the controller to the plant. The unreliable network contains a fading channel and an additive white Gaussian noise (AWGN) channel. We consider two types of feedback control: state feedback and output feedback, and fundamental limitations are obtained for regulation performance, respectively. The optimal regulation performance limitations can be obtained by the limiting steady state solution of its associated algebraic Riccati equation (ARE). Finally, some simulation results are given to illustrate the obtained results.

Furthermore, the obtained results of this paper can be easily extended to the continuous-time case. When the networked control system contains the nondeterministic or hybrid switching, the issue of performance limitation also deserves to be studied furthermore.

\section{Competing Interests}

The authors declare that they have no competing interests.

\section{Acknowledgments}

This work is partially supported by the National Natural Science Foundation of China (61503133, 61374171, 51374107, and 51577057), by Major State Basic Research Development Program (973) subproject (61325309), by the Research Foundation of Education Bureau of Hunan Province (15C0548), by the Province Natural Science Foundation of Hunan under Grants 2016JJ6043 and 14JJ3110, and by the Postdoctoral Science Foundation of China under Grant 2016M592449.

\section{References}

[1] X.-W. Jiang, B. Hu, Z.-H. Guan, X.-H. Zhang, and L. Yu, “Best achievable tracking performance for networked control systems with encoder-decoder," Information Sciences, vol. 305, pp. 184195, 2015

[2] X.-S. Zhan, J. Wu, T. Jiang, and X.-W. Jiang, "Optimal performance of networked control systems under the packet dropouts and channel noise," ISA Transactions, vol. 58, pp. 214-221, 2015.

[3] X.-S. Zhan, X.-X. Sun, T. Li, J. Wu, and X.-W. Jiang, "Optimal performance of networked control systems with bandwidth and coding constraints," ISA Transactions, vol. 59, pp. 172-179, 2015.

[4] Y. Li, J. Chen, E. Tuncel, and W. Su, "MIMO control over additive white noise channels: stabilization and tracking by LTI controllers," IEEE Transactions on Automatic Control, vol. 61, no. 5, pp. 1281-1296, 2016.

[5] X. Dong, Y. Zhou, Z. Ren, and Y. Zhong, "Time-varying formation control for unmanned aerial vehicles with switching interaction topologies," Control Engineering Practice, vol. 46, pp. 26-36, 2016.

[6] Z. W. Liu, X. Yu, Z. H. Guan et al., "Modulated intermittent control in consensus of multi-agent systems," IEEE Transactions on Systems, Man, and Cybernetics: Systems, 2016.

[7] M.-F. Ge, Z.-H. Guan, B. Hu, D.-X. He, and R.-Q. Liao, "Distributed controller-estimator for target tracking of networked robotic systems under sampled interaction," Automatica, vol. 69, pp. 410-417, 2016.

[8] X. Dong, Z. Shi, G. Lu, and Y. Zhong, "Formation-containment analysis and design for high-order linear time-invariant swarm systems," International Journal of Robust and Nonlinear Control, vol. 25, no. 17, pp. 3439-3456, 2015.

[9] X. Dong and G. Hu, "Time-varying formation control for general linear multi-agent systems with switching directed topologies," Automatica, 2016.

[10] S.-I. Azuma and T. Sugie, "Dynamic quantization of nonlinear control systems," IEEE Transactions on Automatic Control, vol. 57, no. 4, pp. 875-888, 2012.

[11] F. Li, P. Shi, L. Wu, M. V. Basin, and C.-C. Lim, "Quantized control design for cognitive radio networks modeled as nonlinear semi-markovian jump systems," IEEE Transactions on Industrial Electronics, vol. 62, no. 4, pp. 2330-2340, 2015.

[12] H. Yan, F. Qian, H. Zhang, F. Yang, and G. Guo, " $H_{\infty}$ fault detection for networked mechanical spring-mass systems with incomplete information," IEEE Transactions on Industrial Electronics, vol. 63, no. 9, pp. 5622-5631, 2016.

[13] Q. Liu, Z. K. Yang, and G. H. Duan, "Improved congestion control algorithm in wide bandwidth and long delay network," Applied Mechanics and Materials, vol. 462-463, pp. 997-1000, 2014.

[14] C. Huang, Y. Bai, and X. Liu, " $H_{\infty}$-state feedback control for a class of networked cascade control systems with uncertain delay," IEEE Transactions on Industrial Informatics, vol. 6, no. 1, pp. 62-72, 2010.

[15] X. Luan, P. Shi, and F. Liu, "Stabilization of networked control systems with random delays," IEEE Transactions on Industrial Electronics, vol. 58, no. 9, pp. 4323-4330, 2011.

[16] D. Liberzon, "Finite data-rate feedback stabilization of switched and hybrid linear systems," Automatica, vol. 50, no. 2, pp. 409420, 2014.

[17] A. J. Rojas, "Signal-to-noise ratio fundamental constraints in discrete-time linear output feedback control," Automatica, vol. 47, no. 2, pp. 376-380, 2011. 
[18] L. Shi and L. Xie, "Optimal sensor power scheduling for state estimation of Gauss-Markov systems over a packet-dropping network," IEEE Transactions on Signal Processing, vol. 60, no. 5, pp. 2701-2705, 2012.

[19] Y. Zou, J. Lam, Y. Niu, and D. Li, "Constrained predictive control synthesis for quantized systems with Markovian data loss," Automatica, vol. 55, pp. 217-225, 2015.

[20] Z.-H. Guan, B. Hu, M. Chi, D.-X. He, and X.-M. Cheng, "Guaranteed performance consensus in second-order multiagent systems with hybrid impulsive control," Automatica, vol. 50, no. 9, pp. 2415-2418, 2014.

[21] Z.-H. Guan, X.-S. Zhan, and G. Feng, "Optimal tracking performance of MIMO discrete-time systems with communication constraints," International Journal of Robust and Nonlinear Control, vol. 22, no. 13, pp. 1429-1439, 2012.

[22] Z.-H. Guan, C.-Y. Chen, G. Feng, and T. Li, "Optimal tracking performance limitation of networked control systems with limited bandwidth and additive colored white Gaussian noise," IEEE Transactions on Circuits and Systems I: Regular Papers, vol. 60, no. 1, pp. 189-198, 2013.

[23] Z.-H. Guan, B. X. Wang, and L. Ding, "Modified tracking performance limitations of unstable linear SIMO feedback control systems," Automatica, vol. 50, no. 1, pp. 262-267, 2014.

[24] H. Okajima and T. Asai, "Tracking performance limitation for 1-DOF control systems using a set of attainable outputs," SICE Journal of Control, Measurement, and System Integration, vol. 8, no. 5, pp. 348-353, 2015.

[25] N. C. Martins, M. A. Dahleh, and J. C. Doyle, "Fundamental limitations of disturbance attenuation in the presence of side information," IEEE Transactions on Automatic Control, vol. 52, no. 1, pp. 56-66, 2007.

[26] N. C. Martins and M. A. Dahleh, "Feedback control in the presence of noisy channels, 'Bode-like', fundamental limitations of performance," IEEE Transactions on Automatic Control, vol. 53, no. 7, pp. 1604-1615, 2008.

[27] J. S. Freudenberg, R. H. Middleton, and J. H. Braslavsky, "Minimum variance control over a Gaussian communication channel," IEEE Transactions on Automatic Control, vol. 56, no. 8, pp. 1751-1765, 2011.

[28] H. Shingin and Y. Ohta, "Disturbance rejection with information constraints: performance limitations of a scalar system for bounded and Gaussian disturbances," Automatica, vol. 48, no. 6, pp. 1111-1116, 2012.

[29] J. Hu and G. Feng, "Distributed tracking control of leaderfollower multi-agent systems under noisy measurement," Automatica, vol. 46, no. 8, pp. 1382-1387, 2010.

[30] N. Elia, "Remote stabilization over fading channels," Systems and Control Letters, vol. 54, no. 3, pp. 237-249, 2005.

[31] N. Xiao, L. Xie, and L. Qiu, "Feedback stabilization of discretetime networked systems over fading channels," IEEE Transactions on Automatic Control, vol. 57, no. 9, pp. 2176-2189, 2012.

[32] E. I. Silva and S. A. Pulgar, "Performance limitations for singleinput LTI plants controlled over SNR constrained channels with feedback," Automatica, vol. 49, no. 2, pp. 540-547, 2013.

[33] R. Penrose, "A generalized inverse for matrices," Mathematical Proceedings of the Cambridge Philosophical Society, vol. 51, no. 3, pp. 406-413, 1955.

[34] M. Athans, "The matrix minimum principle," Information and Control, vol. 11, no. 5-6, pp. 592-606, 1967. 


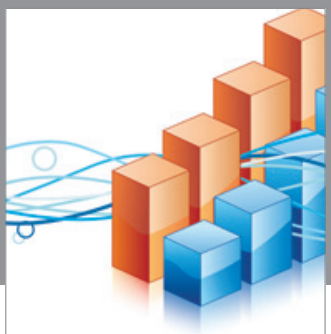

Advances in

Operations Research

vatem alat4

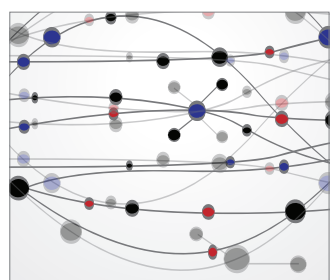

\section{The Scientific} World Journal
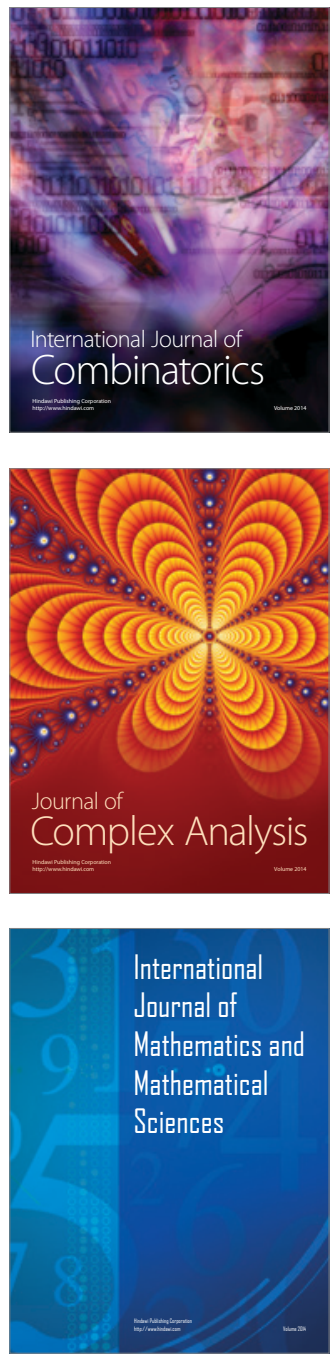
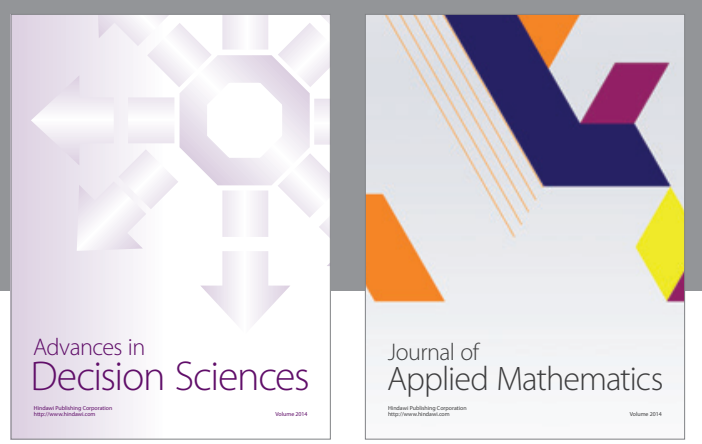

Algebra

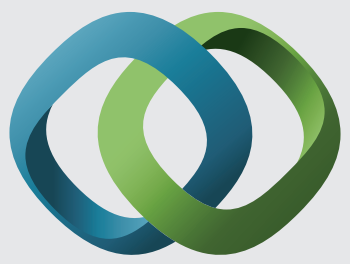

\section{Hindawi}

Submit your manuscripts at

http://www.hindawi.com
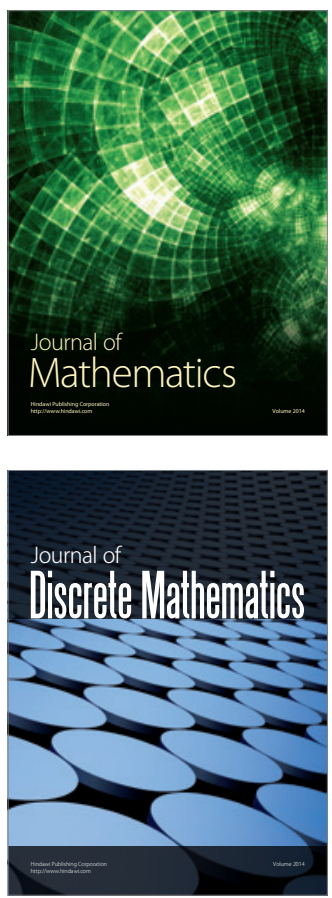

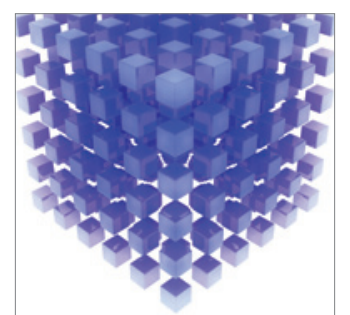

Mathematical Problems in Engineering
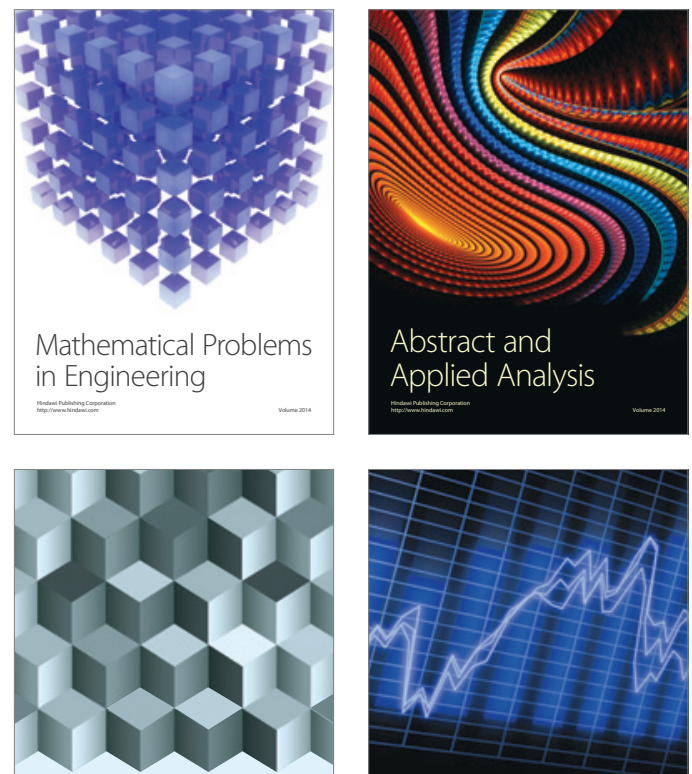

Journal of

Function Spaces

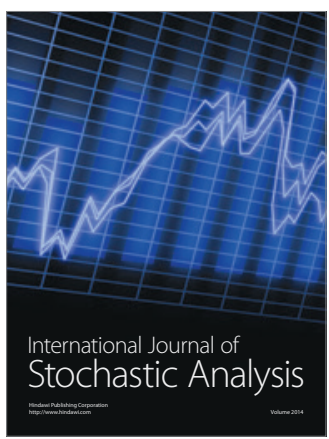

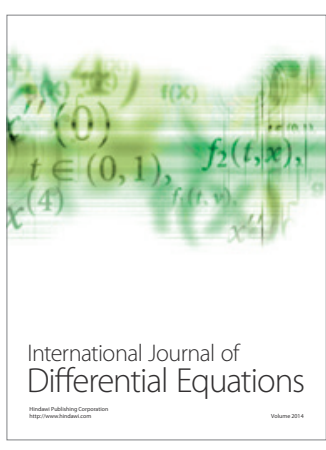
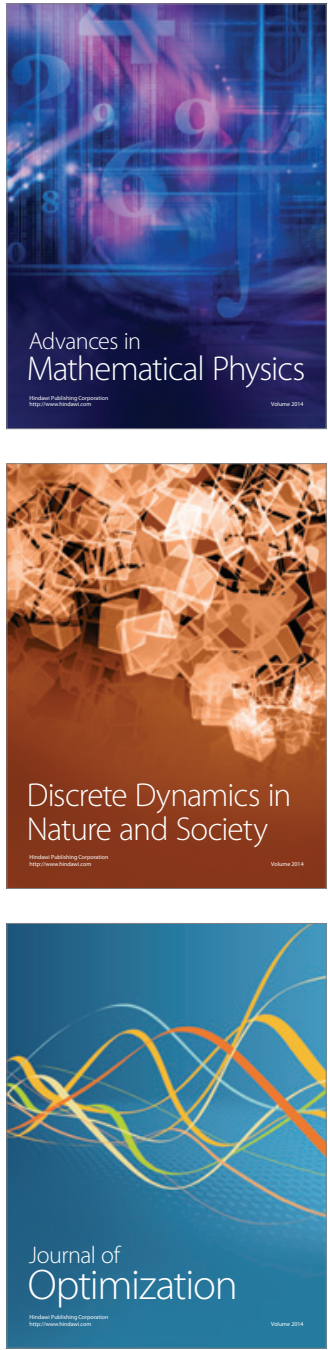